\title{
Validación en español del test TYM para cribado de demencia en una población argentina*
}

\author{
Spanish Validation of the TYM Test for Dementia \\ Screening in an Argentine Population
}

Recibido: enero 3 de 2012 | Revisado: marzo 19 de 2013 | Aceptado: abril 27 de 2013

\author{
DANIEL SERRANI ** \\ Universidad Nacional de Rosario, Santa Fe, Argentina
}

doi:10.11144/Javeriana.UPSY13-1.vetc

Para citar este artículo: Serrani, D. (2014). Validación en español del test TYM para cribado de demencia en una población argentina. Universitas Psychologica, 13(1), 265-284. doi:10.11144/Javeriana. UPSY13-1.vetc

Investigación financiada por la Secretaría de Ciencia y Técnica. Universidad Nacional de Rosario.

** Profesor Departamento de Ciencias Biológicas. Facultad de Psicología de la Universidad Nacional de Rosario. Riobamba 250 Bis, 2000 Rosario, Santa Fe, Argentina. Fax: (54-341) 860224. E-mail: danielserrani@argentina.com

\section{RESUMEN}

El objetivo del presente trabajo fue validar y determinar los puntos de corte de un test neurocognitivo rápido de cribado de demencia llamado Test Your Memory ([TYM]; Test Your Memory - Evalúa Tu Memoria), traducido y modificado para adaptarlo a las características de una muestra de sujetos procedente de la ciudad de Rosario. Se compararon los resultados entre pacientes con demencia y controles normales apareados por edad y sexo. Para la validación y selección de puntos de corte del TYM se lo comparó con otros test estandarizados testigos (Test de Lobo o MEC, Addenbrook's Cognitive Test en Español-Revisado o ACE-R y Test de Pfeiffer o PFAQ) en dos muestras de sujetos formadas por 50 pacientes dementes y 100 controles normales. Los pacientes con demencia completaron el TYM con puntaje promedio de 37/50 mientras que los controles puntuaron 47/50. La correlación con los otros tests fue muy buena. Un puntaje $<40 / 50$ tuvo sensibilidad de $84 \%$ (95\% CI 82.2-88.7\%) y especificidad de 95\% (95\% CI 92.1-96.9\%) en demencia. Los puntos de corte se establecieron usando curvas ROC. El TYM detectó $97 \%$ de pacientes con demencia mientras que MEC y ACE$\mathrm{R}$ detectaron $78 \%$. El valor predictivo negativo y positivo del TYM con un punto de corte de $<40$ fue de $95 \%$ y $45 \%$, respectivamente para una prevalencia de demencia de $5 \%$. Este punto de corte se redujo a 39/50 para el caso de sujetos con bajo nivel de escolaridad ( $<6$ años de estudios). El TYM es simple y económico, se completó rápida y sencillamente por los pacientes y controles y constituyó una herramienta de cribado válida para la detección de demencia. Sin embargo se previene de su uso indiscriminado sin control por agentes sanitarios o clínicos, quienes deben estar a cargo de la puntuación e interpretación de los resultados.

Palabras clave autor

Validación, cribado demencia, memoria, deterioro cognitivo, salud primaria, test neurocognitivo.

Palabras clave descriptores

Psicología neurocognitiva, demencia, desarrollo.

\section{A B S T R A C T}

The aim of this study is the validation and identification of cut-off scores for a fast neuro-cognitive test, called TYM (Test Your Memory) which goal is dementia screening. It was translated and adapted to meet a sample profiles that dwell in Rosario. Results between patients and normal controls matched for age and gender were compared. To establish cut-off points the TYM test was compared against other standardized well-known test (Test de Lobo or MEC, Addenbrook Cognitive Test in Spanish -Revised or ACE-R and Pfeiffer Test or PFAQ) in two samples of subjects with 50 demented patients and 100 normal controls each one. Patients with dementia completed the 
TYM with a mean score of 37/50 and controls scored 47/50. Correlation with the other tests was good. A score of $<40 / 50$ had a sensibility of $84 \%$ (95\% CI 82.2-88.7\%) and specificity of $95 \%$ (95\% CI 92.1-96.9\%) in dementia. Cut-off scores were settled using ROC curves. TYM detected $97 \%$ of dementia patients while MEC and ACE-R detected 78\%. Positive and negative predictive value of the TYM with a cut-off score of $<40$ was $45 \%$ y $95 \%$ respectively, with a dementia prevalence rate of 5\%. This cut-off score was reduced to 39/50 for those subjects with low level education ( $<6$ years of education). The TYM reveals as a simple, low cost test. Patients and controls completed the test fast and easily and it stands as a valid tool for dementia screening. A prevention must be made, however, against using it with no control by sanitary agents or clinics, who must be in charge of scoring and interpretation of the test results.

Keywords author

Validation, screening, dementia, memory, cognitive impairment, primary health, neurocognitive test.

Keywords plus

Neurocognitive Psychology, Dementia, Development.

El envejecimiento de la población es una característica demográfica que adquiere cada vez mayor importancia (Nitrini et al., 2009). En una investigación reciente que incluyó datos de 6 países latinoamericanos, la prevalencia global de demencia en los adultos mayores ( $\geq 65$ años) fue de $7.1 \%$ (95\% CI: 6.8-7.4), asimilándose a los datos de los países desarrollados. Sin embargo, la incidencia en sujetos relativamente más jóvenes (65-69 años) fue mayor en los estudios de Latinoamérica -siendo la demencia de tipo Alzheimer la causa más común-, lo que podría estar relacionado con menores niveles educacionales y reducida reserva cognitiva, razones de la emergencia más temprana de signos clínicos de demencia. Por otra parte, entre los años 2000 y 2025, casi 57 millones de adultos mayores se incorporarán a los 41 millones existentes; entre 2025 y 2050 este incremento será de 86 millones de personas. Los países de mayor población en la región (Brasil y México, junto a Colombia, Argentina, Venezuela y Perú) concentrarán la mayor parte de este aumento, pero en los países más pequeños este aumento también será significativo, especialmente a partir de 2025. Entre el 2025 y el 2050, entre un quinto y un cuarto del incremento de la población adulta mayor se producirá en estos países. Esta situación plantea un enorme reto ya que la proporción de la población de 60 años y más pasará del $8 \%$ que se registró en el 2000 a $14.1 \%$ que se verificará en el 2025 y a un $22.6 \%$ para el año 2050.

Dadas estas cifras, se deduce que los trastornos mentales constituyen un asunto prioritario y representan un desafío para los países latinoamericanos en términos de diagnóstico precoz y, cuando se cronifican (Kohn et al., 2005) costos económicos crecientes. El deterioro de las condiciones de salud física y mental que caracteriza al envejecimiento se acompaña de múltiples enfermedades crónicas, incluyendo la demencia, que por sus características representa una amenaza angustiante para los pacientes, una carga gravosa para los sistemas de salud y en todos los casos un alto costo económico y social. Las estimaciones de frecuencia y prevalencia efectuadas en otros países arrojan cifras entre 1 y 13\% (Andersen-Ranberg, 2013; Crimmins \& Beltrán, 2011). Esta dispersión se debe a diferencias en la franja de edad de las muestras estudiadas y los criterios diagnósticos usados para la categorización. Según el GBD 2000, la prevalencia puntual de las demencias en conjunto es del 0.6\%; en mayores de 60 años es del $5 \%$ para varones y $6 \%$ para mujeres, se duplica cada 5.1 años después de los 65 y supera el 35\% en mayores de 95 años. La incidencia no difiere por sexos, pero la mayor longevidad femenina se traduce en mayor número de mujeres con demencia. Se estima que alrededor de 24 millones de personas en el mundo tienen un diagnóstico de demencia y las personas afectadas se duplican cada 20 años (Adelman, Blanchard, Rait, Leavey \& Livingston, 2011; Prince et al., 2013). Se requiere la medición de prevalencia de demencia para determinar necesidades y demandas sanitarias y para ello se deben realizar tamizajes en poblacionales locales.

Como primer paso para el diagnóstico, se debe detectar un deterioro cognitivo a través de test, escalas de valoración funcional, examen clínico y de laboratorio y estudio de neuroimágenes. Esta batería completa implica un alto coste no solo económico sino de tiempo, además de requerir baremos adecuados para descartar falsos negativos y positivos. Por esto, se necesitan pruebas sencillas, rápidas, con adecuada sensibilidad y especificidad para detectar los casos probables de demencia en atención primaria. En los test de función cogni- 
tiva se consideran su estructura diagnóstica y los factores culturales, idiomáticos y geográficos para determinar su validez, estandarizar su aplicación y seleccionar puntos de corte para la población local con vistas a obtener conclusiones fidedignas y comparar los resultados con otras poblaciones (Nitrini et al., 2009). Si bien hay numerosos test cognitivos actualmente disponibles, pocos cumplen los requisitos críticos para ser utilizados por agentes sanitarios no especializados como: tiempo de administración mínimo, evaluación de un conjunto razonable de funciones cognitivas y sensibilidad para detección precoz de demencia. Idealmente, un test de tamizaje debería reunir las siguientes condiciones: rápido y simple, fácil de realizar, seguro, aceptable, económico, aplicable a todos los sujetos (equidad), válido (sensible y específico), fiable, no sesgado, adaptado culturalmente, validado en la población en la que se aplica, evaluado correctamente.

Entre los factores que contribuyen a limitar el diagnóstico de demencia en los países de escasos recursos figuran los problemas de acceso a los servicios sanitarios (Gómez, Curcio, \& Duque, 2009; Fratiglioni, 2012), la escasez de recursos humanos especializados (Petersen, Lund \& Stein, 2011), bajo nivel de conocimiento sobre esta problemática en la población e incluso entre los profesionales (Kakuma et al., 2011; Saxena, Thornicroft, Knapp \& Whiteford, 2007), dificultad para utilizar instrumentos consensuados, económicos, rápidos y adecuados para todos los niveles educativos (Ashford et al., 2007; Mayeux et al., 2011). Teniendo en cuenta estas circunstancias se tradujo un instrumento neurocognitivo destinado al tamizaje de demencia llamado Test Your Memory ([TYM]; Brown, Pengas, Dawson, Brown, \& Clatworthy, 2009a, 2009b), traducido al español como Evalúa tu Memoria, y se realizó su validación para una muestra poblacional de la ciudad de Rosario, contrastándolo con el Mini-Examen Cognoscitivo de Lobo ([MEC]; Lobo, Escoba, Exquerra \& Sevar-Díaz, 1979; Uysal-Bozkir, Parlevliet \& de Rooij, 2013); el Pfeiffer Short Portable Mental Status Questionnaire ([PFAQ]; Pfeiffer, 1975) y el Addenbrooks Cognitive Examination-Revised en español ([ACE-R]; Mioshi, Dawson, Mitchell, Arnold. \& Hodges, 2006).

\section{Método}

\section{Materiales}

Todos los pacientes fueron sometidos a una evaluación neuropsicológica que fue realizada por dos neuropsicólogas independientes, quienes aplicaron la siguiente batería de test: 1) subtest de Memoria Lógica del Weschler Memory Scale, 2) Test de aprendizaje auditivo verbal de Rey, 3) Trail Making Test A y B, 4) Figura Compleja de Rey inmediata y demorada a los 30 minutos, 5) Test de denominación de Boston, 6) Wisconsin Card Sorting Test, 7) Test de Sustitución Dígito-Símbolo del Weschler Adult Intelligence Scale III y 8) el Digit Span numérico y verbal. Un neurólogo independiente y ciego a la investigación llevó a cabo el examen neurológico mediante entrevista estructurada para el diagnóstico de demencia, examen físico completo, aplicación de la Cambridge Behavioural Inventory (Roth, 1986), el Neuropsychiatric Inventory (Cheng, Kwok \& Lam, 2012), la sección B del test CAMDEX (Rabin et al., 2009) y los criterios del DSM IV-TR (American Psychiatric Association [APA], 2000), el NINCDS-ARDRA (McKhann et al., 2011) para confirmación del diagnóstico de demencia, sin hacer referencia a los resultados del TYM.

El diagnóstico se basó en un consenso entre el neurólogo, las neuropsicólogas y el investigador principal, tomando en consideración los resultados obtenidos en las distintas pruebas diagnósticas sin tener en cuenta el TYM. Para detección del deterioro cognitivo se utilizaron los tests MEC, ACE-R y PFAQ además del TYM (Tabla 1)

El MEC es un test breve que evalúa la orientación en tiempo y espacio, registro, memoria reciente, atención, nominación y comprensión del lenguaje, habilidad visuo-espacial, omitiendo abstracción y juicio, con validez y confiabilidad comprobadas. Su mejor puntaje es de 33 y con un punto de corte de 23/24 permite distinguir entre pacientes normales y con deterioro cognitivo. La sensibilidad para demencia oscila entre 76 y 100\% y la especificidad entre 78 y 100\% (Roth et al., 1986). El nivel educativo influye en el resultado del test y su validez, siendo esta una situación frecuente entre los adultos mayores que 
TABLA 1

Valores psicométricos de las pruebas utilizadas para contrastar el TYM

\begin{tabular}{lccc}
\hline & ACE-R & MEC & PFAQ \\
\hline Validez convergente & $r=0.952$ & $r=0.857$ & $r=0.795$ \\
Confiabilidad & $\alpha$ Cronbach $=0.918$ & $\alpha$ Cronbach=0.832 & $\alpha$ Cronbach=0.809 \\
Punto de corte & 76 & 23 & 6 \\
Sensibilidad & $0.89(79.2-94.5)$ & $0.81(71.3-85.1)$ & $0.86(67.4-89.9)$ \\
Especificidad & $0.87(74.3-95.2)$ & $0.84(72.1-87.4)$ & $0.83(73.4-88.2)$ \\
Valor predictivo positivo & $52.4(45.3-76.3)$ & $42.1(36.7-56.7)$ & $46.3(35.9-65.7)$ \\
Valor predictivo negativo & $90.3(85.3-95.3)$ & $81.4(75.9-84.7)$ & $89.2(81.4-91)$ \\
Valor global & $55.4(44.3-76.8)$ & $43.7(34.6-56.4)$ & $51.9(49.6-69.9)$ \\
\hline
\end{tabular}

Fuente: elaboración propia.

exhiben bajos niveles de escolaridad, resultando en falsos diagnósticos de deterioro cognitivo (Appels \& Scherder, 2010). En tanto, el PFAQ evalúa 10 actividades que van desde manejar su propio dinero, comprar ropas y comestibles, calentar agua, apagar la cocina, preparar una comida, estar al tanto de la actualidad, entender un programa de radio o TV, recordar compromisos, manejar medicamentos, volver a la casa, saludar a sus amigos o permanecer en casa en forma segura. Tiene un punto de corte de $<6$ para diferenciar entre demencia y control normal. El ACE-R evalúa 6 dominios cognitivos: orientación (10), atención (8), memoria (35), fluencia verbal (14), lenguaje (28) y habilidades visuo-espaciales (5). El puntaje máximo obtenible es 100.

Por su parte, el TYM está formado por una serie de 10 tareas distribuidas en dos hojas con espacios en blanco que el paciente debe completar con sus respuestas. La habilidad del paciente para completar el test es la 11 1 tarea. El test incluye orientación (10 puntos), habilidad para copiar una frase (2 puntos), memoria semántica (3 puntos), cálculo (4 puntos), fluencia verbal (4 puntos), similitudes (4 puntos), nominación (5 puntos), habilidades visuoespaciales (2 tareas, total 7 puntos) y recuerdo de la oración copiada (6 puntos). La habilidad de completar el test se puntúa también (5 puntos), dando un resultado total de 50 puntos. Los resultados para cada una de las tareas se colocan en la hoja impresa y el puntaje total se calcula con base en la suma de cada una de las tareas parciales. Para evitar errores de la puntuación, se provee una hoja adicional con las instrucciones para la puntuación y el cálculo de la suma total. En el caso del TYM se realizaron dos traducciones independientes (del inglés al español) y otras dos traducciones inversas (del español al inglés) siendo las traductoras ciegas al original. A continuación se remitieron las traducciones inversas a las traductoras originales quienes no encontraron diferencias entre ambas versiones, determinando la viabilidad lingüística del instrumento.

Luego de contar con la versión traducida al español se realizó una experiencia piloto con una muestra reducida de sujetos normales, a partir de la cual se introdujeron modificaciones en algunos puntos, basadas en la frecuencia de uso local de ciertos términos y conceptos, sin modificar el sentido original. En las preguntas de orientación se dejaron como versión final: iquién es el/la presidente?, ien qué año comenzó la Guerra de Malvinas?; en la pregunta de copia se eligió: "los buenos ciudadanos usan zapatos lustrados"; en la pregunta de nombrar 4 criaturas que empiecen con la letra $S$ se la reemplazó por la siguiente: "nombrar 4 animales que empiecen con la letra P". En las preguntas de semejanzas se eligieron las siguientes versiones: ien qué se parecen un tomate y una papa? y ien que se parecen un puma y un león?; en la tarea visuo-espacial se eligió la letra oculta "M". La tarea de nominación de partes de la figura no sufrió modificaciones (ver Apéndice).

Se seleccionaron 50 pacientes ( 20 hombres y 30 mujeres) con trastorno cognitivo, derivados de centros de atención primaria de la ciudad de Rosario, a los cuales se les administró el TYM en primer lugar 
para luego ser sometidos a evaluación neurológica y neuropsicológica completas, administración del ACE-R en español, el MEC, el PFAQ, exploración con neuroimágenes (TAC, RM y SPECT de fotón único) y análisis de sangre para descartar causas secundarias de demencia. De los 50 pacientes, 32 tenían un diagnóstico de Demencia Degenerativa Primaria tipo Alzheimer probable, 10 tenían diagnóstico de Demencia Vascular, 4 tenían Demencia con Cuerpos de Lewy y 3 tenían Demencia FrontoTemporal de acuerdo a los criterios diagnósticos ya establecidos (Irish, Graham, Graham, Hodges \& Hornberger, 2012; Salloway, 2012). La severidad de la demencia fue clasificada mediante la escala Clinical Dementia Rating [(CDR)]; Hughes, Berg, Danziger, Coben \& Martin, 1982) y su amplitud de rango fue de 0.5 a 1 . La CDR permite evaluar el estadio de evolución de la demencia por medio de una escala de 5 puntos en la cual CDR 0 denota ausencia de alteración cognitiva, 0.5 expresa demencia muy leve, CDR 1 indica demencia leve, CDR 2 muestra demencia moderada y CDR 3 señala demencia severa. El puntaje se obtiene de un conjunto de observaciones estandarizadas incluyendo 6 dominios como memoria, orientación, juicio, resolución de problemas, desempeño en hogar y habilidades domésticas, cuidados personales y asuntos comunitarios, valorando la habilidad cognitiva del paciente para funcionar en cada una de esas áreas, independientemente de las limitaciones o fragilidad física que pudieran limitar sus desempeños. En cada caso se obtiene la información a partir del propio paciente y de una fuente colateral. El puntaje del CDR global se deriva a partir de reglas publicadas (O’Bryant et al., 2010).

No se incluyeron en el estudio los pacientes con trastornos del estado de ánimo, que fue evaluado mediante la escala de Yesavage abreviada de 15 puntos (valores de exclusión > 6) (Mitchell, Bird, Rizzo \& Meader, 2010; Yesavage, Brinkm \& Rose, 1983), ni tampoco los que presentaban déficits neurológicos, deterioro motor o sensorial, antecedentes de consumo de drogas o traumatismos cráneo-encefálicos. Los controles, 100 personas en total, fueron seleccionados por edad y sexo para aparejarlos a los pacientes y se reclutaron entre los

TABLA 2

Composición demográfica de la muestra (apareados por edad y sexo)

\begin{tabular}{ccccccc}
\hline \multirow{2}{*}{ Grupos de edad } & \multicolumn{2}{c}{ Hombres } & \multicolumn{2}{c}{ Mujeres } & \multicolumn{2}{c}{ Totales } \\
\cline { 2 - 6 } & $\mathrm{N}$ & $\%$ & $\mathrm{~N}$ & $\%$ & $\mathrm{~N}$ & $\%$ \\
\hline $60-69$ & $30(10)$ & 20 & $45(15)$ & 30 & $75(25)$ & 50 \\
$70-79$ & $24(8)$ & 16 & $30(10)$ & 20 & $54(18)$ & 36 \\
$80-89$ & $6(2)$ & 4 & $15(5)$ & 10 & $21(7)$ & 14 \\
Total & $60(20)$ & 40 & $90(28)$ & 60 & $150(48)$ & 100 \\
\hline Edad media & $77.8 \pm 9.29$ & \multicolumn{2}{c}{$78.8 \pm 8.23$} & $78.1 \pm 7.81$ \\
\hline
\end{tabular}

Nota. Porcentaje sobre el total de cada sexo y sobre el total general. Entre paréntesis figura el número de sujetos con demencia en cada grupo. Grupos de edad en años.

Fuente: elaboración propia.

TABLA 3

Composición de la muestra por escolaridad

\begin{tabular}{ccccc}
\hline \multirow{2}{*}{ Estado cognitivo } & \multicolumn{3}{c}{ Escolaridad } & \multirow{2}{*}{ Totales } \\
\cline { 2 - 4 } & $>6$ años & $<6$ años & 100 \\
Controles $(\mathrm{n})$ & 70 & 30 & 50 \\
\hline Demencia $(\mathrm{n})$ & 30 & 20 & 150 \\
\hline Total $(\mathrm{n})$ & 100 & 50 & \\
\hline
\end{tabular}

$N=$ número

Fuente: elaboración propia. 
acompañantes y familiares de los pacientes, excluyendo a los que tenían historia de enfermedades neurológicas, problemas de memoria, daño cerebral, consumo de drogas o trastornos afectivos, todos los cuales dieron su consentimiento informado. Los controles tenían entre 60 y 89 años de los cuales 90 (60\%) eran mujeres (Tabla 2). La composición por escolaridad se observa en la Tabla 3.

En primer término, se administró el TYM traducido y modificado a los 100 controles distribuidos en grupos de edad, para evaluación de confiabilidad del instrumento y obtención de datos normativos. Luego, se extrajeron las medias para TYM global y subtest en los diferentes grupos etarios, realizando una comparación entre las mismas para poner en evidencia efectos sobre los resultados del TYM debidos a la edad. Posteriormente, se administró el TYM a los 50 pacientes y luego se les hizo completar el ACE-R, el MEC y el PFAQ, tanto a pacientes como a controles, comparando los resultados entre ellos en subtotales y totales del TYM, mediante ANOVA de mediciones repetidas para comparaciones múltiples. Los resultados de TYM se compararon con ACE-R y MEC usando coeficientes Rho de Spearman y tau de Kendall. La consistencia interna del TYM se evaluó con $\alpha$ de Cronbach.

Con los resultados del TYM en pacientes y controles se construyó una curva ROC y se calcularon los valores predictivos positivos y negativos, punto de corte, sensibilidad y especificidad. Se realizó una curva ROC para TYM de sujetos con menos de 6 años de escolaridad, identificando el punto de corte con mejor balance entre especificidad y sensibilidad. Se graficaron las curvas ROC con resultados de ACE-R, MEC y PFAQ. Finalmente, se efectuó una comparación de curvas ROC de todos los test utilizados para tamizado de demencia. La especificidad del TYM se calculó mediante el porcentaje de los 50 pacientes con demencia que podían ser identificados con puntos de corte $<40$ para TYM,$<23$ para MEC, $<6$ para PFAQ y $<86$ para ACE-R. El acuerdo entre evaluadores se midió con coeficientes Kappa y peso lineal, y se realizó comparando las evaluaciones de dos entrevistadores independientes para la muestra de pacientes y controles, usando la misma hoja de puntuación. Uno de los evaluadores fue un médico con experiencia en demencias y el otro un MAP (médico de atención primaria) sin experiencia concreta en el tema de demencias que recibió un entrenamiento de 1 hora. Los test fueron puntuados de manera independiente.

El análisis estadístico de los registros se efectuó con el programa Medcalc (versión 11.2.1) y el paquete estadístico EPIDAT versión 2.1 para Windows (EPIDAT Versión 2.1). Se calculó la sensibilidad y especificidad para cada ítem del TYM y los resultados se plotearon en curvas ROC (Receiver Operating Characteristics) para determinar puntos de corte según el mejor balance entre sensibilidad y especificidad. Las curvas ROC permiten mostrar de manera gráfica las modificaciones que se producen cuando mejora la sensibilidad o la especificidad, usando diferentes puntos de corte, siendo muy usadas para comparar diferentes test diagnósticos. El mejor balance se anota en el ángulo superior izquierdo de la curva y se selecciona como punto de corte para el test. Mientras mejor es el test, más se acerca la curva al ángulo superior izquierdo del gráfico (Jack et al., 2011). Se calcularon sensibilidad, especificidad, valores predictivos positivo (la probabilidad de que un individuo con resultado positivo tenga la patología) y negativo (la probabilidad de que un individuo con resultado negativo no tenga la patología), teniendo en cuenta diferentes prevalencias teóricas, además de los límites de confianza para los puntos de corte seleccionados. Se estudió, mediante regresión lineal múltiple, la relación entre la puntuación del TYM como variable dependiente y las variables de regresión sexo, edad y años de educación.

\section{Resultados}

El TYM se completó de manera rápida y satisfactoria, usando un tiempo promedio de 2 a 5 minutos tanto para controles como para pacientes, y requirió en la mayoría de los casos una mínima asistencia por parte el entrevistador. Se midió la consistencia interna para pacientes y controles en el conjunto de subítems del TYM mediante el coeficiente $\alpha$ de Cronbach (Browner et al., 1992; Cronbach, 1951), consistente en establecer la correlación promedio 
entre ítems; siendo su valor de 0.8. Los resultados del TYM para los controles por rangos de edad fueron los siguientes: entre 60/69 años el promedio del TYM fue de 49/50 (95\% CI de 48 a 49, $\chi^{2}=250.318$ $g l=15 ; p<0.0001)$; entre 70/79 años el promedio fue de 48/50 (95\% CI de 47 a 49, $\chi^{2}=224.995 \mathrm{gl}$ $=14, p<0.0001$ ); finalmente, entre 80/89 años el promedio fue de 43/50 ( $\chi^{2}=295.110 \mathrm{gl}=130$, $p<0.0001)$. Las diferencias entre los dos primeros grupos $(F=1.9455 ; p=0.05)$ resultaron menores con respecto al último grupo etario $(F$ test $(g l=98)$ $=4.16 ;$ CI $95 \% 3.81$ a 4.5, $p<0.0001)$, con un efecto significativo de la edad sobre los subtest del TYM especialmente para la copia $(F=4.36 ; p<0.001)$, memoria semántica $(F=2.68$; $p=0.007)$, cálculo $(F=3.55 ; p=0.006)$, fluencia $(F=4.75$, $p=0.009)$, similitudes $(F=4.66 ; p=0.005)$, memoria anterógrada $(F=3.34 ; p<0.003)$ y ayuda requerida $(F=9.11 ; p<0.004)$. El TYM global para sujetos con escolaridad $<6$ años arrojó un valor promedio de $39.013 \pm 3.7$ (CI 95\% 38.421 a 39.605). La puntuación del TYM no mostró dependencia con el sexo ni con la edad, pero sí con los años de educación, con un coeficiente de regresión de 1.529 (CI 95\% de 0.875-2.101). El sumario general de todos los test neurocognitivos aplicados se muestra en la Tabla 4.

El coeficiente de correlación de Pearson de TYM con MEC fue de 0.678 (CI 95\% de 0.58 a $0.756, p<0.0001$ ); de TYM con PFAQ fue de -0.578 (CI 95\% - 0.676 a $-0.46, p<0.0001$ ) y de TYM con ACE-R fue de 0.597 (CI 95\% 0.498 a $0.679, p<0.0001$ ). Una vez aplicados los tests TYM, ACE-R, MEC y PFAQ se construyeron curvas ROC para diferentes puntos de corte. Los resultados se muestran en las Figuras 1, 2, 3 y 4.

El área bajo la curva ROC para diferenciar entre controles y dementes fue de 0.992 [CI 95\% 0.962 a $1 ; p($ área $=0.5)<0.0001$ ], el mejor balance entre sensibilidad y especificidad se obtiene alrededor del punto de corte de $<40$, con una sensibilidad de $84 \%$ (CI 95\% 82.6-89.7) y una especificidad de 95\% (CI 95\% 93.9-98.9); Likelihood Ratio+ = 9.51 (CI 95\% 8.6-10,5); Likelihood Ratio- $=0.09$ (0.008-0.1), Valor Predictivo+ = 45 (CI 95\% 42-47), Valor Predictivo- $=95$ (CI 95\% = 93.6-97.7). Si se baja el punto de corte a $<39$ se eleva la especificidad a $96 \%$ pero baja la sensibilidad a $83 \%$. Sin embargo, cuando se separa el grupo por nivel educacional, se observa que en el grupo de menos de 6 años de educación el área bajo la curva ROC es de 0.969 [CI 95\% 0.927 a 0.99, p (área $=0.5$ ) $<0.0001]$, con el mejor punto de corte en $<39$ con una sensibilidad de $84 \%$ (CI $95 \% 80.2$ a 87.7 ) y especificidad de $94 \%$ (CI 85\% 92.1 a 96.9), LR+ = 22.5 (CI 95\% 20.3 a 24.9) y LR- $=0.1$ (CI 95\% 0.03 a 1.7), $\mathrm{VP}+=$ 46 (CI 95\% 43.5 a 48.7) y VP- = 94 (CI 95\% 92.1 a 96.4). Estos puntos de corte se establecieron de acuerdo con una prevalencia teórica de enfermedad de 5\% (Tabla 5).

Comparando la capacidad del TYM con MEC, ACE-R y PFAQ para detectar los 50 pacientes con demencia, se observa que con un punto de corte de $<40$ para TYM se detecta el $95 \%$, con punto de corte $<23$ en MEC se detecta 79.59\% (CI 95\% 65.7 a $89.8 ; \mathrm{LR}+=4.9, \mathrm{LR}-=0.04 ; \mathrm{VP}+=44.9$, VP. $=92)$, con punto de corte de $<86$ se detecta el $94.78 \%(\mathrm{CI} 95 \% 91.41$ a $97.7, \mathrm{LR}+=14.05$,

TABLA 4

Resumen general de medias y CI de test neurocognitivos utilizados

\begin{tabular}{lcccc}
\hline & \multicolumn{2}{c}{ Control } & \multicolumn{2}{c}{ Pacientes con demencia } \\
\cline { 2 - 5 } & Media (DE) & $95 \% \mathrm{CI}$ & Media (DE) & $95 \% \mathrm{CI}$ \\
\hline MEC & $29.8 \pm 2.36$ & $29.4-30.3$ & $21.14 \pm 2.45$ & $20.4-21.8$ \\
PFAQ & $3.05 \pm 0.18$ & $2.62-3.57$ & $13.9 \pm 0.4$ & $12.3-15.9$ \\
TYM & $45.2 \pm 3.16$ & $44.6-45.9$ & $37.7 \pm 2.85$ & $35.9-39.5$ \\
ACE-R & $85.9 \pm 2.23$ & $85.5-86.4$ & $79.9 \pm 2.06$ & $79.3-80.5$ \\
\hline
\end{tabular}

MEC = MiniExamen Cognoscitivo de Lobo, PFAQ = Pfeiffer Short Portable Questionnaire, TYM = Test your Memory (Evalúa tu Memoria), ACE-R = Addenbrooke Cognitive Examination-Revised.

Fuente: elaboración propia. 


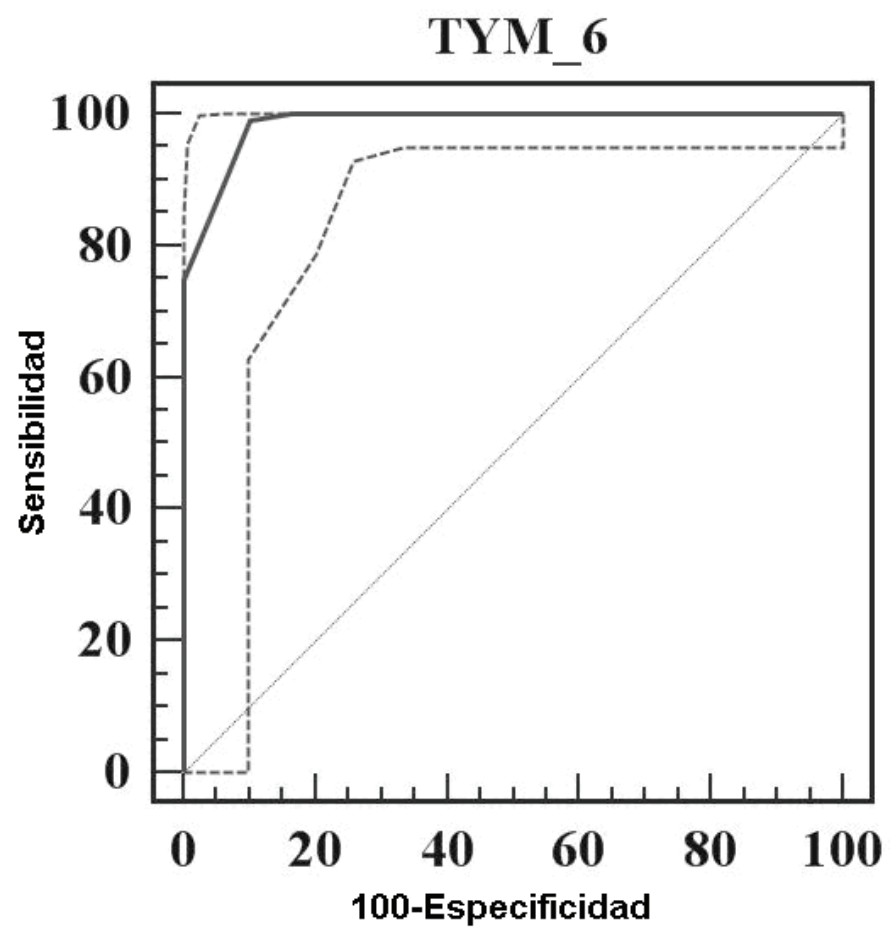

Figura 1. Curva ROC en TYM con < 6 años escolaridad (TYM 6).

Fuente: elaboración propia.

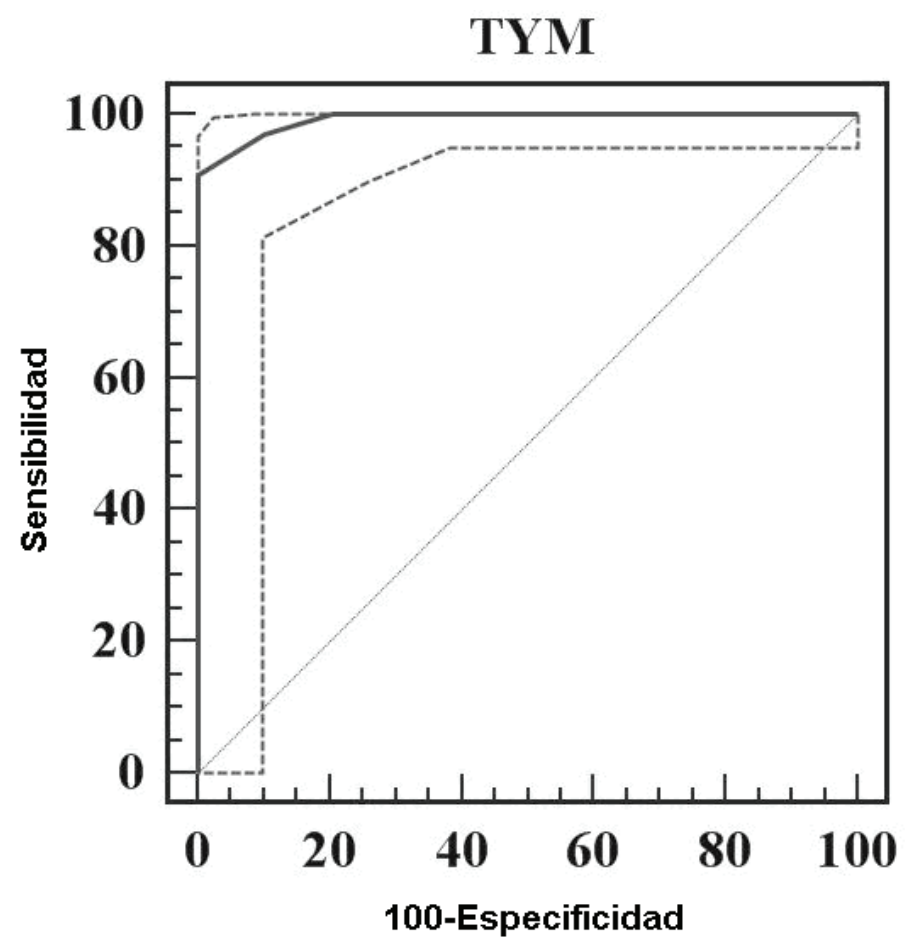

Figura 2. Curva ROC para TYM Global.

Fuente: elaboración propia. 


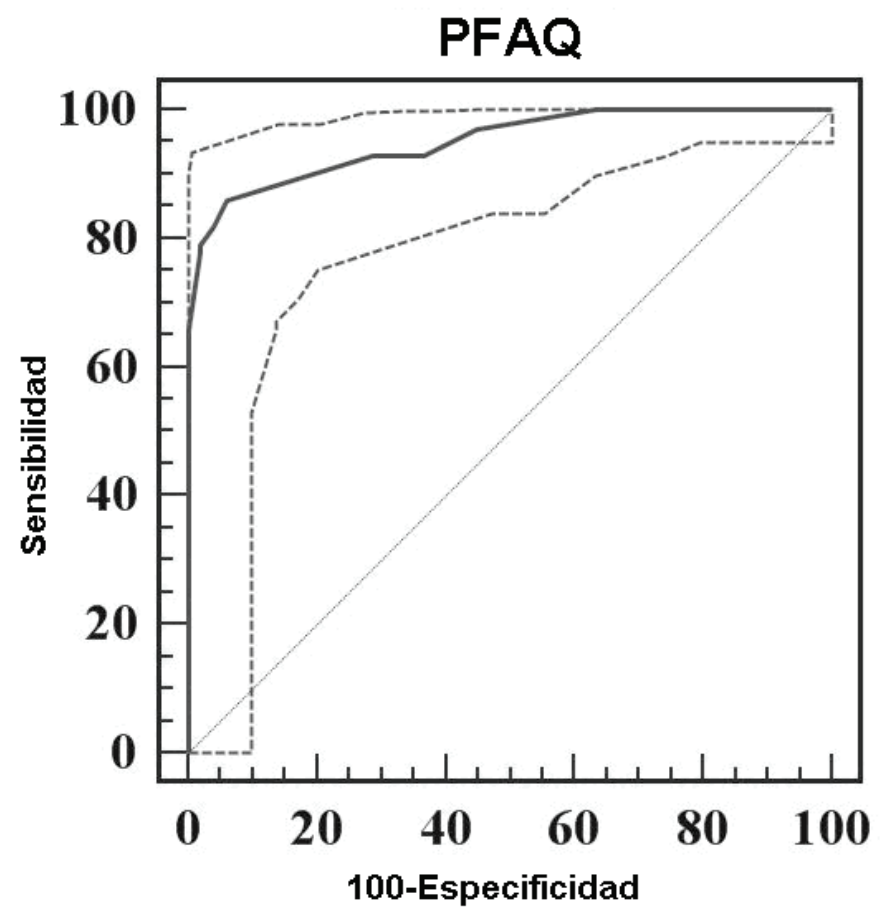

Figura 3. Curva ROC para PFAQ.

Fuente: elaboración propia.

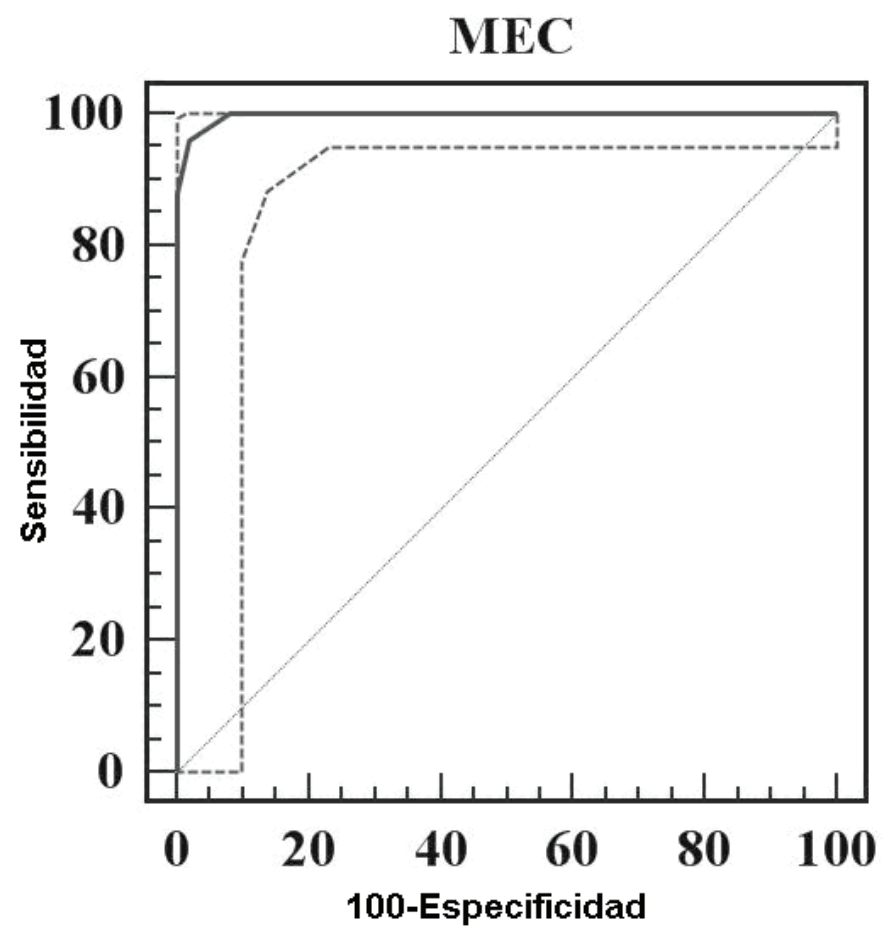

Figura 4. Curva ROC para MEC.

Fuente: elaboración propia

| Universitas Psychologica | V.13 | No.1 | enero-marzo | 2014 | 
TABLA 5

Sensibilidad, especificidad y VP $+y$-para distintos puntos de corte del TYM con escolaridad $<y>$ de 6 años

\begin{tabular}{|c|c|c|c|c|c|c|c|c|c|c|}
\hline \multirow{4}{*}{$\begin{array}{l}\text { Punto de } \\
\text { corte }\end{array}$} & & & & & \multicolumn{6}{|c|}{ prevalencia } \\
\hline & & & & & \multicolumn{2}{|c|}{$5 \%$} & \multicolumn{2}{|c|}{$10 \%$} & \multicolumn{2}{|c|}{$15 \%$} \\
\hline & \multicolumn{2}{|c|}{ Especificidad (\%) } & \multicolumn{2}{|c|}{ Sensibilidad (\%) } & & & & & & \\
\hline & $<6$ años & $>6$ años & $<6$ años & $>6$ años & VP+ & VP- & $\mathrm{VP}+$ & VP- & $\mathrm{VP}+$ & VP- \\
\hline$<38$ & 95 & 97 & 83 & 80 & 47 & 93 & 63 & 94 & 83 & 95 \\
\hline$<39$ & 94 & 96 & 84 & 83 & 46 & 94 & 60 & 95 & 82 & 96 \\
\hline$<40$ & 93 & 95 & 86 & 84 & 45 & 95 & 57 & 96 & 81 & 97 \\
\hline$<41$ & 90 & 92 & 87 & 85 & 44 & 96 & 56 & 97 & 80 & 98 \\
\hline$<42$ & 87 & 90 & 93 & 90 & 43 & 97 & 55 & 98 & 79 & 99 \\
\hline$<43$ & 86 & 87 & 95 & 93 & 42 & 98 & 53 & 99 & 73 & 99 \\
\hline$<44$ & 83 & 84 & 98 & 96 & 40 & 99 & 51 & 99 & 71 & 99 \\
\hline
\end{tabular}

$\mathrm{VP}+=$ valor predictivo positivo, $\mathrm{VP}-=$ valor predictivo negativo.

Fuente: elaboración propia.

LR- = 0.15); con punto de corte de $<6$ en PFAQ se detecta $93.88 \%$ (CI 95\% 90.1 a 96.7, LR+ = 38.71, $\left.\mathrm{LR}_{-}=0.21 ; \mathrm{VP}+=42.8, \mathrm{VP}-=79.6\right)$ y con punto de corte < 39 en TYM para escolaridad menor a 6 años se detecta el 94\% (CI 95\% 83.1 a 98.7; $\left.\mathrm{LR}+=14.54, \mathrm{LR}_{-}=0.12, \mathrm{VP}+=46, \mathrm{VP}-=94\right)$ La distribución del grupo control y grupo demencia de acuerdo con un punto de corte de $<40$ se ve en la Figura 5. Se compararon las áreas bajo las curvas ROC para el TYM, MEC ACE-R para evaluar la capacidad discriminativa de los mismos entre el grupo control y el de demencia. De la curva comparativa surge un área de 0.992 para el TYM, de 0.934 para el ACE-R y 0.911 para el MEC, con una diferencia estadísticamente significativa entre los mismos $(p<0.01)$, indicativa de una mayor sensibilidad y

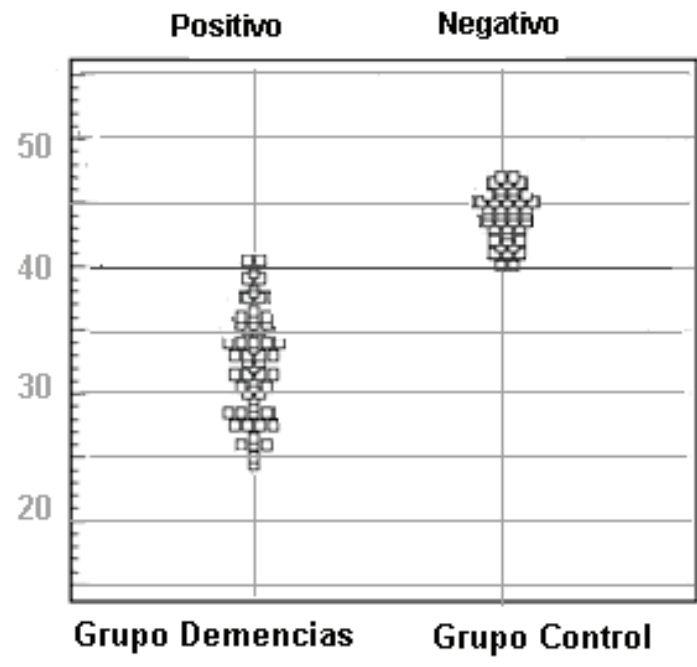

$<40$

Sensibilidad: 84

\section{Especificidad: 95}

Figura 5. Distribución de casos controles y pacientes con demencia con un punto de corte $<40$ para TYM $>6$ años de escolaridad, para una sensibilidad de 84 y especificidad de 95. Los puntos de la izquierda representan los pacientes con demencia, los falsos negativos son los puntos ubicados por encima de la línea de punto de corte de 40. Los puntos de la derecha representan los controles; los falsos positivos son los puntos ubicados por debajo de la línea de punto de corte de 40.

Fuente: elaboración propia. 
especificidad del TYM sobre los restantes test para el diagnóstico de demencia.

\section{Discusión}

Conforme a la validación del TYM se detecta un deterioro cognitivo con un puntaje de $<40$, y tomando en consideración una menor escolaridad el punto de corte se ajusta a $<39$. Con una adecuada sensibilidad y especificidad se detectan $95 \%$ de los casos de demencia, con una sensibilidad del 84\%. Todos los sujetos pudieron completar el test de manera sencilla y relativamente rápida, manteniendo un promedio de $45 / 50$ para controles normales (49/50 para grupo de edad de 60-69, 48/50 para grupo de edad de $70-79$ y $43 / 50$ para grupo de edad de 80-89) y $37 / 50$ para demencia, es decir 8 puntos por debajo de los controles normales. Los resultados en subtest del TYM declinan con la edad. Se mantuvo un ligero sesgo femenino en pacientes y controles, reproduciendo una tendencia habitual en el envejecimiento, sin representar fuente de diferencias en los resultados del TYM. Además se mantuvo la exclusión sobre controles y pacientes que exhibieran síntomas de depresión o consumo de drogas. La baja escolaridad produjo un efecto en los resultados del TYM, con un promedio de $38.93 \pm 1.7$ en las puntuaciones (CI 95\% de 36.6 a 40.73; rango intercuartiles $=5$ ) y un punto de corte de $<39$.

El análisis de los resultados de los subtest del TYM en controles y demencia muestra deterioro en este último grupo, exhibiendo las menores diferencias en copia de oración, nominación, orientación, y similitudes y mostrando un patrón característico de demencia en la baja ejecución sobre las pruebas que requieren el uso de memoria anterógrada, memoria semántica, fluencia y tareas visuo-espaciales, aunque las diferencias no fueron estadísticamente significativas. En relación con las diferencias entre los controles sanos de menos y más de 80 años, se observa una declinación en el grupo de más edad (media $=48.5 \pm 0.74$ en $<$ 80 años vs. $M=43 \pm 1.41$ en $>80$ años), pero aun así permanece por encima de las diferencias globales entre controles sanos y dementes, con promedios de resultados del TYM de 45.2 y 35.7 , respectivamente. Por otra parte, no hay diferencias significativas debidas a la edad en el grupo de dementes $(\mathrm{M}=38.38 \pm 1.68<80$ años vs. $\mathrm{M}=36.57 \pm 1.71>80$ años). Esto sugiere que el TYM es útil para detectar los pacientes ancianos con demencia, con una sensibilidad y especificidad altas. Una puntuación de $<40$, detecta el 95\% de los casos de demencia; una puntuación $<42$ detecta $92 \%$ de los pacientes.

La prevalencia de la demencia en la población general a menudo impone un límite en la especificidad de los test cognitivos, teniendo en cuenta que los sujetos afectos de demencia tienen un pobre insight de su afección. Los evaluadores fueron independientes pero conocían el diagnóstico o la condición de control de los sujetos examinados. No obstante, dado que cada sujeto completaba la hoja del test de manera individual, estos evaluadores tuvieron una mínima influencia en el resultado final, la cual se redujo aún más por el uso de una ficha de puntuación estándar.

La correlación del TYM y Test de Lobo (MEC) fue buena, teniendo en cuenta que este último es un instrumento confiable y ampliamente utilizado para la evaluación cognitiva de pacientes con demencia. Sin embargo, no evalúa un rango completo de dominios cognitivos ya que cuantifica básicamente 10 funciones intelectuales como orientación, memoria de fijación, memoria reciente, concentración y atención, nominación, órdenes verbales y escritas, escritura y construcción (copia de dibujo), pero tiene un sesgo negativo hacia las funciones temporal y parietal del hemisferio dominante. Evalúa de manera satisfactoria la orientación, pero tiene un efecto techo en los test de memoria y lenguaje que resultan demasiado simples y ofrece un solo punto para las tareas visuo-espaciales. Estas desventajas lo ponen en una situación de debilidad para la detección de los casos de demencia leves, con una tasa $79.59 \%$ de detección con un punto de corte de < 23 en el presente estudio.

Estos datos son concordantes con otros estudios (Mathuranath, Nestor, Berrios, Rakowicz \& Hodges, 2000; Stein et al., 2012). Tiene un tiempo de administración de 10 a 20 minutos de acuerdo 
con informes hospitalarios, que es considerado demasiado largo (Milian, 2012; Torisson, Minthon, Stavenow \& Londos, 2012). En el presente estudio el TYM cumple con el criterio de brevedad, pudiendo ser completado en 2 a 5 minutos en la misma sala de espera, analizado por el evaluador en un promedio de 2 minutos. Se puede observar de manera directa al paciente completando el test, lo que colabora en el diagnóstico. El TYM evalúa 11 tareas explorando más dominios cognitivos que el MEC, con menos sesgo hacia las funciones del lenguaje del hemisferio dominante. Por otra parte, las tareas de lenguaje y memoria exhiben una mayor carga hacia la capacidad cognitiva del sujeto. La inclusión de dos tareas visuo-espaciales agrega 7/50 puntos al resultado global permitiendo una mejor discriminación de la demencia con respecto a los cuadros con predominio amnésico. El MEC también tiene desventajas en cuanto a la localización del examinado, ya que los pacientes desorientados espacialmente pueden puntuar 4 puntos más en su domicilio que en el hospital o la clínica. El MEC resulta parcialmente inadecuado para el monitoreo de la evolución del paciente, a pesar de ser ampliamente utilizado con esta finalidad (van Schalkwyk, Botha \& Seedat, 2012). El TYM tiene una mayor amplitud $(35 / 50 \Delta 15)$ en rango de resultados que el MEC (20/33 $\Delta 13)$.

En relación con el ACE-R, el TYM tiene ventaja en el tiempo de administración (2-5 minutos vs. 20 minutos), aunque ambos evalúan un número similar de dominios y son comparables en la tasa de detección de demencia (94.78\% del ACE-R vs. 95\% del TYM) y con respecto al PFAQ tiene mejor capacidad de detección (93.88\% del PFAQ vs. 95\% del TYM), compartiendo con este último la brevedad de administración pero explorando un número sensiblemente inferior de dominios cognitivos.
En resumen, el TYM ha demostrado ser un instrumento útil para la administración rápida en la consulta con un sistema de puntuación confiable con buen acuerdo inter-raters (Tabla 6), sin necesidad de tener experiencia previa en el caso de los evaluadores, que pueden entrenarse brevemente en menos de media hora.

En el presente estudio, las modificaciones realizadas en la traducción y en los conceptos explorados sirvieron para ajustarlo a las características locales de la población, sin modificar su validez interna y congruencia. El valor predictivo negativo de 95 con punto de corte $<40$ indica que en grupos no seleccionados un puntaje superior hace muy improbable la presencia de demencia, lo cual confiere al TYM buen valor de cribado para problemas de memoria en población general. Con puntos de corte mayores a 41 el valor predictivo negativo se eleva a 96 con prevalencia de enfermedad de $5 \%$, y con un punto de corte de 43 el valor predictivo negativo se eleva a 99 con una prevalencia de enfermedad de 15\%, permitiendo excluir prácticamente el diagnóstico de demencia en grupos no seleccionados.

Dado que en la presente investigación se excluyeron los pacientes con déficits neurológicos severos o deterioro sensorial o motor, es posible que en una población con estos problemas se requiera algún tipo de ayuda para completar los formularios, en cuyo caso los resultados no serían comparables. En todo caso, esto queda como una posible limitación del estudio. El valor predictivo positivo con punto de corte de $<40$ es de 45,57 y 81 para prevalencia de demencia del 5, 10 y $15 \%$, respectivamente en población seleccionada, lo cual indica que TYM no puede ser usado en exclusividad para el diagnóstico de demencia en población general no seleccionada. Por otra parte, disminuyendo el punto de corte a

TABLA 6

Acuerdo de evaluación entre jueces para los test TYM por MAP y especialista en demencia

\begin{tabular}{cccc}
\hline & & \multicolumn{2}{c}{ Correlación $\left(r^{2}\right)$} \\
\cline { 3 - 4 } & Score General $(\mathrm{DE})(/ 50)$ & MAP & Especialista \\
\hline MAP & $40.23(4.7)$ & - & - \\
Especialista & $40.97(4.1)$ & $0.99(0.87)$ & - \\
\hline
\end{tabular}

$\mathrm{MAP}=$ médico de atención primaria. Especialista $=$ médico con experiencia en demencias.

Fuente: elaboración propia. 
$<39$ se pueden discriminar satisfactoriamente los pacientes con menor nivel de escolaridad de aquellos con demencia, con una especificidad del 94\%, un valor predictivo negativo de 94 y valor predictivo positivo de 46.

El TYM evalúa memoria y lenguaje además de tareas visuo-espaciales de modo menos simple que el MEC, evitando así la relativa insensibilidad de este último en la detección de los estadios tempranos de demencia o los casos muy leves. El TYM puede ser completado por el paciente de manera relativamente autónoma, mientras espera en la sala de recepción, de modo que se limita la influencia del médico o del familiar/acompañante, aunque no se puede impedir de manera absoluta la colaboración en situaciones no controladas. De todas maneras, cualquier ayuda recibida se minimiza con el uso de formularios preimpresos. El TYM explora un número mayor de dominios cognitivos que el MEC o el PFAQ, y por ello registra menos sesgo hacia las funciones del hemisferio dominante representadas por el lenguaje, con la inclusión de tareas visuoespaciales.

Con respecto al ACE-R, el TYM se administra en menos tiempo, aunque registra igual número de dominios cognitivos. Con respecto a la muestra de población participante, a diferencia de la investigación original, se reclutaron pacientes con diferentes tipos de demencia, sin que esto influyera significativamente en los valores de respuesta al TYM y los puntos de corte seleccionados, lo cual le resta valor discriminativo con respecto a demencia de tipo Alzheimer (Haubois et al., 2013), es decir que no se presenta como instrumento apto para el diagnóstico diferencial de los diversos tipos de demencias en sus estadios iniciales.

Una posible explicación de esta limitación es el grado leve de demencia de los sujetos participantes (CDR 0.5 a 1), lo que confiere al TYM mayor utilidad para el tamizaje inicial de los trastornos demenciales y cognitivos antes que para el diagnóstico diferencial de estos últimos. La misma aclaración es válida para la diferenciación entre trastornos demenciales y trastornos del estado de ánimo que pueden cursar con alteraciones en el rendimiento cognitivo. El test ha sido diseñado para ser completado por el paciente de manera individual y autónoma, con una mínima instrucción por parte del agente sanitario, quien por otra parte solo requiere una breve formación previa, ahorrando de este modo un tiempo valioso para el profesional. No obstante su puntuación e interpretación quedan a cargo del clínico quien en caso de puntuaciones anormales puede derivar el caso al equipo interdisciplinario, asegurando una investigación más detallada durante el tiempo que sea necesario y sin excluir en este caso el recurso a otros instrumentos neurocognitivos más detallados. De modo tal que queda claro que el test no está diseñado para el autodiagnóstico y en ningún caso queda en manos del paciente la interpretación de los resultados, ya que al utilizarse en población general pueden surgir situaciones de falsos positivos cuyo manejo debe ser definido por el clínico o agente sanitario actuante (Black, 2009; Kendall, Pilling, Pettinari \& Whittington, 2004).

Con respecto al subtest de nominación del TYM se escogió la letra P como descriptor categorial basándose en su mayor frecuencia de uso en la población argentina (Butman, Allegri, Harris \& Drake, 2000). Se ha utilizado un punto de corte en el ACE-R de 86 para su comparación con el TYM. Este valor ha sido extraído de estudios anteriores (Sarasola, 2005), pero no se puede afirmar que sea representativo de la población general, tal como aclaran los autores. Esto puede constituir una posible limitación en los resultados comparativos.

\section{Referencias}

Adelman, S., Blanchard, M., Rait, G., Leavey, G. \& Livingston, G. (2011). Prevalence of dementia in African-Caribbean compared with UK-born white older people: Two-stage cross-sectional study. The British Journal of Psychiatry, 199(2), 119-125.

American Psychiatric Association. (2000). Diagnostic and statistical manual of mental disorders (ed. rev.). Washington, DC: Autor.

Andersen-Ranberg, K. (2013). Trends over time in cognitive function and activities of daily living among Danish centenarians. Annual Review of Gerontology and Geriatrics, 33(1), 333-359. 
Appels, B. A. \& Scherder, E. (2010). Review: The diagnostic accuracy of dementia-screening instruments with an administration time of 10 to 45 minutes for use in secondary care: A systematic review. American Journal of Alzheimer's Disease and other Dementias, 25(4), 301-316.

Ashford, J. W., Borson, S., O'Hara, R., Dash, P., Frank, L., Robert, P., Shankle, W. R., et al. (2007). Should older adults be screened for dementia? It is important to screen for evidence of dementia! Alzheimer's $\mathcal{B}$ Dementia, 3(2), 75-80.

Bastide, L., De Breucker, S., Van den Berge, M., Fery, P., Pepersack, T. \& Bier, J. C. (2012). The Addenbrooke's Cognitive Examination revised is as effective as the original to detect dementia in a French-speaking population. Dementia and Geriatric Cognitive Disorders, 34(5-6), 337-343.

Black, S. L. (2009). Too many false positives. BMJ, 339, b2830.

Brown, J., Pengas, G., Dawson, K., Brown, L. A. \& Clatworthy, P. (2009, junio 10). Self administered cognitive screening test (TYM) for detection of Alzheimer's disease: Cross sectional study. BMJ, 338. http://dx.doi.org/10.1136/bmj.b2030

Brown, J., Pengas, G., Dawson, K., Brown, L., Clatworthy, P. (2009). TYM and Alzheimer's disease. Author's resply. BMJ, 339, b2825.

Browner, W., Newman, T., Cummings, S. (1992). Designing a new study: III. Diagnostic tests. In S. Hulley \& S. Cummings (Eds.), Designing clinical research. Baltimore: Williams \& Wilkins.

Bullain, S. S., Corrada, M. M., Shah, B. A., Mozaffar, F. H., Panzenboeck, M. \& Kawas, C. H. (2013). Poor physical performance and dementia in the oldest-old the $90+$ study physical performance and dementia in the oldest-old. JAMA Neurology, 70(1), 107-113.

Butman, J., Allegri, R., Harris, P. \& Drake, M. (2000). Fluencia verbal en español. Datos normativos en Argentina. MEDICINA, 60, 561-564.

Cerami, C., Scarpini, E., Cappa, S. F. \& Galimberti, D. (2012). Frontotemporal lobar degeneration: Current knowledge and future challenges. Journal of Neurology, 259(11), 2278-2286.

Cheng, S. T., Kwok, T. \& Lam, L. C. (2012). Neuropsychiatric symptom clusters of Alzheimer's disease in
Hong Kong Chinese: Prevalence and confirmatory factor analysis of the Neuropsychiatric Inventory. International Psychogeriatrics, 1(1), 1-9.

Crimmins, E. M. \& Beltrán-Sánchez, H. (2011). Mortality and morbidity trends: Is there compression of morbidity? The Journals of Gerontology Series B: Psychological Sciences and Social Sciences, 66(1), 75-86.

Cronbach, L. J. (1951). Coefficient alpha and the internal structure of tests. Psychometrika, 16(3), 297-333. de Vries, K., Brooker, D. J. \& Smith, P. (2012). Dementia skills and competencies for primary care liaison: A model for improving identification and timely diagnosis. Primary Health Care Research $\mathcal{E}$ Development, 1(1), 1-10.

EPIDAT. Análisis Estadístico de datos epidemiológicos. Versión 2.1 para Windows.

Francis, N. J. (2012). Assessment tools for geriatric patients with delirium, mild cognitive impairment, dementia, and depression. Topics in Geriatric Rehabilitation, 28(3), 137-147.

Fratiglioni, L. (2012). Epidemiology of Alzheimer's disease and current possibilities for prevention. Acta Neurologica Scandinavica, 94(S165), 33-40.

Freedman, V. A., Spillman, B. C., Andreski, P. M., Cornman, J. C., Crimmins, E. M., Kramarow, E., et al. (2013). Trends in late-life activity limitations in the United States: An update from five national surveys. Demography, 50(2), 661-671.

Gomez, F., Curcio, C. L. \& Duque, G. (2009). Health care for older persons in Colombia: A country profile. Journal of the American Geriatrics Society, 57(9),1692-1696.

Haubois, G., Decker, L., Annweiler, C., Launay, C., Allali, G., R Herrmann, F. \& Beauchet, O. (2013). Derivation and validation of a short form of the Mini-Mental State Examination for the screening of dementia in older adults with a memory complaint. European Journal of Neurology, 20(3), 588-590.

Hughes, C. P., Berg, L., Danziger, W. L., Coben, L. A. \& Martin, R. L. (1982). A new clinical scale for the staging of dementia. British Journal of Psychiatry, $140,566-572$.

Irish, M., Graham, A., Graham, K. S., Hodges, J. R. \& Hornberger, M. (2012). Differential impairment of 
source memory in progressive versus non-progressive behavioral variant frontotemporal dementia. Archives of Clinical Neuropsychology, 27(3), 338-347. Ismail, Z., Rajji, T. K. \& Shulman, K. I. (2010). Brief cognitive screening instruments: An update. International Journal of Geriatric Psychiatry, 25(2), 111-120.

Jack, C. R., Albert, M. S., Knopman, D. S., McKhann, G. M., Sperling, R. A., Carrillo, M. C. \& Phelps, C. H. (2011). Introduction to the recommendations from the National Institute on Aging-Alzheimer's Association workgroups on diagnostic guidelines for Alzheimer's disease. Alzheimer's and Dementia, 7(3), 257-262.

Kakuma, R., Minas, H., van Ginneken, N., Dal, M., Desiraju, K., Morris, J., et al. (2011). Human resources for mental health care: Current situation and strategies for action. Lancet, 378(9803), 1654-1663.

Karzmark, P., Llanes, S., Tan, S., Deutsch, G. \& Zeifert, P. (2012). Comparison of the frontal systems behavior scale and neuropsychological tests of executive functioning in predicting instrumental activities of daily living. Applied Neuropsychology, 19(2), 81-85.

Kendall, T., Pilling, S., Pettinari, C. \& Whittington, C. (2004). Clinical guidelines in mental health I: The National Collaborating Centre for Mental Health. Psychiatric Bulletin, 28(5), 156-159.

Kohn, R., Levav, I., Caldas de Almeida, J. M., Vicente, B., Andrade, L., Caraveo-Anduaga, J. J., et al. (2005). Los trastornos mentales en América Latina y el Caribe: asunto prioritario para la salud pública. Revista Panamericana de Salud Pública, 18(4-5), 229-240.

Lobo, A., Escoba, V., Exquerra, J. \& Sevar-Díaz, A. (1979). El Mini-Examen Cognoscitivo: un test sencillo, práctico, para detectar alteraciones intelectuales en pacientes psiquiátricos. Actas Luso Españolas de Neurología, Psiquiatría y Ciencias Afines, 3(7), 189-202.

Mathew, R. \& Mathuranath, P. S. (2008). Issues in evaluation of cognition in the elderly in developing countries. Annals of Indian Academy of Neurology, 11(2), 82-88

Mathuranath, P. S., Nestor, P. J., Berrios, G. E., Rakowicz, W., Hodges, J. R. (2000). A brief cognitive test battery to differentiate AD and frontotemporal dementia. Neurology, 55(11), 1613-1620.

Mayeux, R., Reitz, C., Brickman, A. M., Haan, M., Manly, J. J., Glymour, M. M., et al. (2011). Operationalizing diagnostic criteria for Alzheimer's disease and other age-related cognitive impairment-Part 1. Alzheimer's 82 Dementia, 7(1), 15-34.

McKhann, G. M., Knopman, D. S., Chertkow, H., Hyman, B. T., Jack, C. R., Kawas, C. H., et al. (2011). The diagnosis of dementia due to Alzheimer's disease: Recommendations from the National Institute on Aging-Alzheimer's Association workgroups on diagnostic guidelines for Alzheimer's disease. Alzheimer's and Dementia, 7(3), 263-269.

Meléndez Moral, J. C., Sanz-Álvarez, T. \& NavarroPardo, E. (2012). Deterioro cognitivo leve: método y procedimiento de clasificación. Anales de Psicología, 28(2), 604-610.

Milian, M., Leiherr, A. M., Straten, G., Müller, S., Leyhe, T. \& Eschweiler, G. W. (2012). The Mini-Cog Versus the Mini-Mental State Examination and the Clock Drawing Test in daily clinical practice: Screening value in a German Memory Clinic. International Psychogeriatrics, 24(5), 766-774.

Mioshi, E., Dawson, K., Mitchell, J., Arnold, R. \& Hodges, J. R. (2006). The Addenbrooke's Cognitive Examination Revised (ACE-R): A brief cognitive test battery for dementia screening. International Journal of Geriatric Psychiatry, 21(11), 1078-1085.

Mitchell, A. J., Bird, V., Rizzo, M. \& Meader, N. (2010). Which version of the geriatric depression scale is most useful in medical settings and nursing homes? Diagnostic validity meta-analysis. American Journal of Geriatric Psychiatry, 18(12), 1066-1077.

Nitrini, R., Bottino, C. M., Albala, C., Custodio Capuñay, N. S., Ketzoian, C., Llibre Rodriguez, J. J., et al. (2009). Prevalence of dementia in Latin America: A collaborative study of population-based cohorts. International Psychogeriatrics, 21(4), 622-630.

O’Bryant, S. E., Lacritz, L. H., Hall, J., Waring, S. C., Chan, W., Khodr, Z. G. \& Cullum, C. M. (2010). Validation of the new interpretive guidelines for the clinical dementia rating scale sum of boxes score in the national Alzheimer's coordinating center database. Archives of Neurology, 67(6), 746 749. 
Olafsdottir, M. \& Marcusson, J. (2012). Diagnosis of dementia at the primary care level. Acta Neurologica Scandinavica, 94(S165), 58-62.

Palacios-Ceña, D., Jiménez-García, R., HernándezBarrera, V., Alonso-Blanco, C., Carrasco-Garrido, P. \& Fernández-de-las-Peñas, C. (2012). Has the prevalence of disability increased over the past decade (2000-2007) in elderly people? A Spanish population-based survey. Journal of the American Medical Directors Association, 13(2), 136-142.

Petersen, I., Lund, C. \& Stein, D. J. (2011). Optimizing mental health services in low-income and middleincome countries. Current Opinion in Psychiatry, 24(4), 318-323.

Pfeiffer, E. (1975). A short portable mental status questionnaire for the assessment of organic brain deficit in elderly patients. Journal of the American Geriatric Society, 23(10), 433-441.

Prince, M., Acosta, D., Ferri, C. P., Guerra, M., Huang, Y., Jacob, K. S. \& Hall, K. S. (2011). A brief dementia screener suitable for use by non-specialists in resource poor settings - the cross-cultural derivation and validation of the brief Community Screening Instrument for Dementia. International Journal of Geriatric Psychiatry, 26(9), 899-907.

Prince, M., Bryce, R., Albanese, E., Wimo, A., Ribeiro, W. \& Ferri, C. P. (2013). The global prevalence of dementia: A systematic review and metaanalysis. Alzheimer's $\mathcal{E}$ Dementia, 9(1), 63-75.

Rabin, L. A., Paré, N., Saykin, A. J., Brown, M. J., Wishart, H. A., Flashman, L. A., et al. (2009). Differential memory test sensitivity for diagnosing amnestic mild cognitive impairment and predicting conversion to Alzheimer's disease. Aging, Neuropsychology, and Cognition, 16(3), 357-376.

Roman, G. C., Tatemichi, T. K., Erkinjuntti, T., Cummings, J. L., Masdeu, J. C., Garcia, J. H., Amaducci, L., et al. (1993). Vascular dementia: Diagnostic criteria for research studies (Report of the NINDSAIREN International Workshop). Neurology, 43, 250-260.

Roth, M., Tym, E., Mountjoy, C. Q., Huppert, F. A., Hendrie, H., Verma, S., et al. (1986). CAMDEX. A standardised instrument for the diagnosis of mental disorder in the elderly with special reference to the early detection of dementia. British Journal of Psychiatry, 149, 698-709.

Salloway, S. (2012). Clinical and pathological examples of Alzheimer's disease, dementia with lewy bodies, and frontotemporal dementia. Medicine and Health-Rhode Island, 95(7), 207-209.

Saraceno, B., Van, O. M., Batniji, R., Cohen, A., Gureje, O., Mahoney, J., et al. (2007). Barriers to improvement of mental health services in low-income and middle-income countries. Lancet, 370(9593), 1164-1174.

Sarasola, D., Calcagno, M., Sabe, L., Crivelli, L., Torralva, T., Roca, M., García-Caballero, A., et al. (2005). El Addenbrooke's Cognitive Examination en español para el diagnóstico de demencia y para la diferenciación entre enfermedad de Alzheimer y demencia frontotemporal. Revista de Neurología, 41(12), 717-721.

Saxena, S., Thornicroft, G., Knapp, M. \& Whiteford, H. (2007). Resources for mental health: Scarcity, inequity, and inefficiency. Lancet, 370(9590), 878889.

Stein, J., Luppa, M., Maier, W., Wagner, M., Wolfsgruber, S., Scherer, M. \& Riedel-Heller, S. G. (2012). Assessing cognitive changes in the elderly: Reliable Change Indices for the Mini-Mental State Examination. Acta Psychiatrica Scandinavica, 126(3), 208-218.

Strydom, A., Hassiotis, A., King, M. \& Livingston, G. (2009). The relationship of dementia prevalence in older adults with intellectual disability (ID) to age and severity of ID. Psychological Medicine, 39(1), 13-21.

Torisson, G., Minthon, L., Stavenow, L. \& Londos, E. (2012). Cognitive impairment is undetected in medical inpatients: A study of mortality and recognition amongst healthcare professionals. BMC Geriatrics, 12(1), 47. doi: 10.1186/1471-2318-12-47 Uysal-Bozkir, Ö., Parlevliet, J. L. \& de Rooij, S. E. (2013). Insufficient cross-cultural adaptations and psychometric properties for many translated health assessment scales: A systematic review. Journal of Clinical Epidemiology, 66(6), 608-618.

Van Schalkwyk, G., Botha, H. \& Seedat, S. (2012). Comparison of 2 dementia screeners, the Test Your Memory Test and the Mini-Mental State Examina- 
VALIDACIÓN EN ESPAÑOL DEL TEST TYM PARA CRIBADO DE DEMENCIA EN UNA POBLACIÓN ARGENTINA

tion, in a primary care setting. Journal of Geriatric Psychiatry and Neurology, 25(2), 85-88.

Yesavage, J. A., Brinkm, T. L. \& Rose, T. L. (1983). Development and validation of a geriatric depression screening scale: A preliminary report. Journal of Psychiatric Research, 17(1), 37-49. 


\section{Apéndice 1}

\section{Test TYM (Evalúa tu Memoria)}

Por favor escriba su nombre completo.

Hoy es:. día

La fecha de hoy es: ........ de. (mes) $20 \ldots . .$.

¿Qué edad tiene? años

¿En qué fecha nació? (día)/ .(mes)/ 20......(año)

10 puntos

Por favor copie la siguiente oración:

Las personas educadas usan zapatos lustrados

2 puntos

¿Quién es el Presidente actual?

¿En qué año comenzó la segunda Guerra Mundial?

3 puntos

Sumas

$20-4=$

$16+17=$

$8 \times 6=$

$4+15-17=$

4 puntos

Por favor haga una lista de 4 animales que empiezan con la letra "p", ejemplo perro

1 p.....................

2 p.....................

$3 \mathrm{p}$

$4 \mathrm{p}$.

4 puntos

¿En qué se parecen un tomate y una papa?

¿En qué se parecen un león y una pantera?

4 puntos

\section{Por favor nombre estas partes}

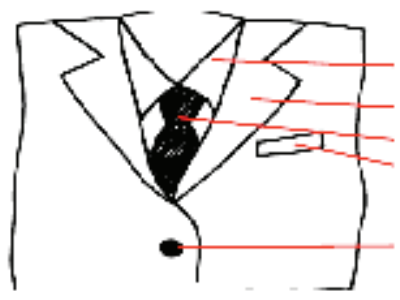

5 puntos 
Por favor una los círculos para formar una letra, ignorando los cuadrados

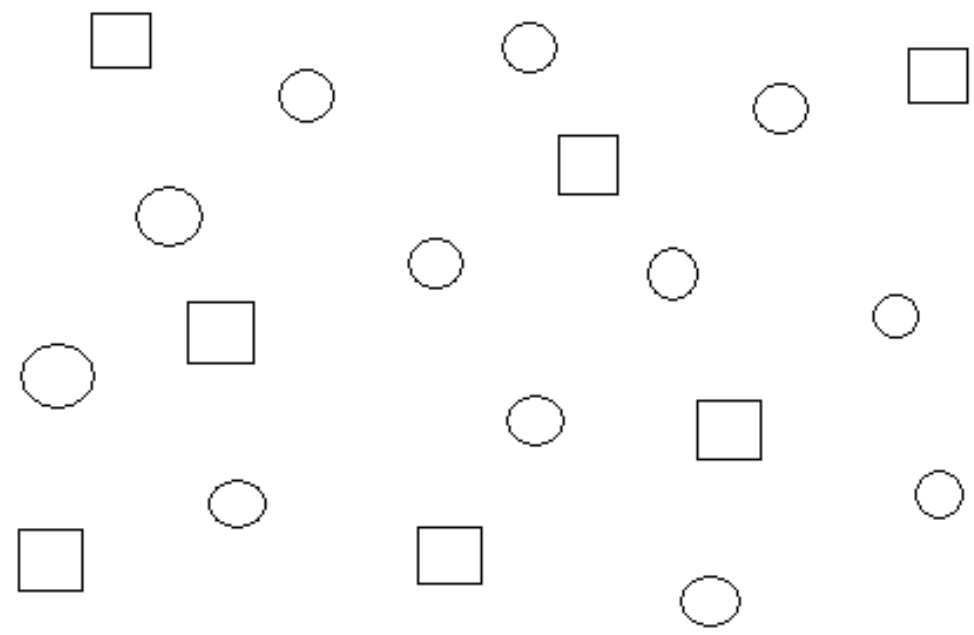

3 puntos

Por favor, utilizando el círculo de abajo, dibuje un reloj, ponga los números del 1 al 12 y ubique las manecillas a las 9.20

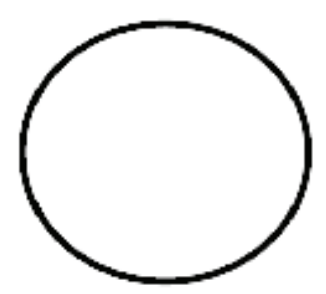

4 puntos

Sin dar vuelta la página escriba la oración que copió hace un momento:

6 puntos

Para el evaluador del test

Ayuda brindada: ninguna/trivial/menor/moderada/mayor

Señale en los apartados si las respuestas son escritas para el paciente

5 puntos

Total/ 50 puntos. 


\section{Apéndice 2}

\section{Puntuación del test TYM (Evalúa tu Memoria)}

Las puntuaciones o abreviaciones no son importantes si se entiende la sentencia (a excepción del punto 2). El mínimo valor en cada pregunta es 0 .

Box 1. 2 puntos para nombre completo, 1 para iniciales /otros errores menores, 1 punto para cada espacio completado correctamente en el resto del box. Si la fecha esta equivocada por un día aun puntúa un punto.

Box 2.2 puntos si todas las palabras están correctas, 1 punto si hay error en 1 palabra, 0 puntos si hay errores en 2 .

Box 3. 1 punto para primer nombre, 1 punto para el apellido. (1982) puntúa 1 punto, total 3.

Box 4. 1 punto para cada suma correcta.

Box 5. Cualquier organismo se considera correcto, sea insecto, pez, pájaro o mamífero.

Box 6. 2 puntos para palabras precisas tales como "hortaliza/vegetal" o "animal/mamífero/cazador/ carnívoro". Respuestas razonables pero menos precisas tales como "alimento", "crece en la tierra”, "cuatro patas" o "fiera" puntúa 1 punto.

Nominación: las respuestas correctas son: cuello/solapa/nudo/bolsillo/botón, puntúan 1 punto cada una. Camisa es aceptable para cuello y saco es aceptable para solapa o bolsillo (pero solo una vez).

Letra M. Si se realiza sin errores puntúa 3 puntos, otra letra formada 2 puntos, si se unen todos los círculos 1 punto.

Reloj. Todos los números puntúa 1, posición correcta de números puntúa 1, manecillas correctas 1 punto cada una.

Oración. Puntúa 1 punto para cada palabra recordada hasta un máximo de 6.

Agregue la puntuación según la cantidad de ayuda que requiera el paciente: Ninguna: agregue + 5, Trivial: agregue +4 , Menor: agregue +3 , Moderada: agregue +2 , Mayor: agregue +1 .

Tilde el Box si las respuestas son escritas por el paciente 